\title{
Study of Bubble Growth in a Multicomponent Mixture at High Pressure
}

\author{
Aitor Amatriain', Ignacio Parra ${ }^{1}$, Gonzalo Rubio ${ }^{1}$ \\ ${ }^{1}$ E.T.S. Ingeniería Aeronáutica y del Espacio / Universidad Politécnica de Madrid \\ Plaza Cardenal Cisneros, 3, 28040, Madrid, Spain \\ a.amatriain@alumnos.upm.es
}

\begin{abstract}
In this work the bubble growth in a multicomponent mixture at high pressure is analysed. This study is highly relevant as it is representative of the change of phase of petroleum in reservoirs. A physical model based in the Navier-Stokes equations complemented by appropriate boundary conditions at the interface between the gas and the liquid phases is presented. The introduced model assumes spherical symmetry to alleviate the computational cost of solving the problem. This computational cost is further reduced by introducing several approximations, which reduce the model to a system of three ordinary differential equations, two parabolic problems and a chemical equilibrium computation. Numerical solutions of this model for a simple mixture are presented.
\end{abstract}

Keywords: Bubble growth, multiphase, multicomponent, chemical equilibrium, petroleum.

\section{Introduction}

The physical problem of the bubble growth has been studied since the middle of the nineteenth century [1]. However, it was at the beginning of the twentieth century when Rayleigh obtained the first conclusions, stating that the radius of a bubble evolves proportionally to time if only inertial effects are considered [2]. Later, the effect of the surface tension, which is important at the beginning of the growth, was also included in this model [3]. With the objective of modeling the thermal effects, Plesset and Zwick [4] included the conservation of energy equation in the aforementioned formulation. Under the assumption of a thin boundary layer and a modified ideal gas model, the authors reduced the thermal problem to a quadrature, concluding that the asymptotic evolution of the radius of a bubble is proportional to the square root of time. This regime is known as diffusive growth period, which arises after two initial phases dominated by inertial and surface tension effects respectively. Several asymptotic techniques have been used to analyse this problem, for example self-similar solutions [5], integral methods [6], or the method of matched asymptotic expansions. In particular, Mikic et al. [7] obtained various interpolation formulas, coupling an initial growth described by Rayleigh solution followed by the Plesset and Zwick thermal model for long times. The development of numerical methods allowed several researchers to propose more accurate models. Forster and Zuber [8] modeled the liquid evaporation at the interphase as an axisymmetric heat source, which allows the computation of the temperature gradient by means of Green's functions at the boundary. Dalle Donne and Ferranti presented a model to study the bubble growth of superheated sodium [9]. These results were verified by Plesset and Prosperetti [10].

The study of bubble growth in multicomponent mixtures can be split into bubble growth in supersaturated mixtures and bubble coalescence in undersaturated mixtures [11]. The last article was the basis of further studies, as the one made by Cable and Evans [12], which considered a finite element model making use of the thermal diffusion equation obtained by Scriven [13]. Recent investigations include the application of asymptotic expansions to the analysis of the Rayleigh-Taylor instability [14], numerical analysis of bubble growth in microchannels [15] and the application of homotopy perturbation methods to bubble growth in gas-hydrates [16].

The present work is focused on the study of bubble growth in multicomponent mixtures at high pressure. This study is highly relevant as it is representative of the change of phase of petroleum in reservoirs, which is an important phenomenon in the oil industry $[17,18]$. This problem has some different features with respect to the aforementioned studies. Firstly, the ratio between gas and liquid density may not be negligible in petroleum. As a result, a usually neglected non-linear term in the energy and species continuity equations is conserved in the model presented here. In addition, both problems are coupled. Secondly, the multicomponent nature of the fluid forces us to solve the chemical equilibrium at the surface of the bubble. This is usually modeled by means of an ideal gas law, that cannot be used here due to the high-pressure constraint. The model presented here tackles this problem by using a cubic equation of state in the chemical equilibrium computation. 
The rest of this work is organised as follows: first, in Section 2 the model is described. Second, in Section 3, results obtained with this model are presented. Finally, in Section 4, the main conclusions are drawn.

\section{Statement of the problem}

\subsection{Navier-Stokes equations}

The model proposed here solves the continuity equation (1), the momentum equation (2), the equation of conservation of energy (3) and the species continuity equation (4) for the incompressible liquid surrounding the bubble. Under the assumptions of spherical symmetry around the bubble, Hirschfelder and Curtiss [19] approximation for the diffusion velocities surrounding the liquid and ideal liquid enthalpy, this model adopts the following form:

$$
\begin{gathered}
\frac{1}{r^{2}} \frac{\partial}{\partial r}\left(r^{2} \rho v\right)=0, \\
\rho \frac{\partial v}{\partial t}+\rho v \frac{\partial v}{\partial r}=-\frac{\partial p}{\partial r}+\frac{1}{r^{2}}\left(r^{2} \mu \frac{\partial v}{\partial r}\right)-2 \mu \frac{v}{r^{2}} \\
\rho c_{l} \frac{\partial T}{\partial t}+\rho v c_{l} \frac{\partial T}{\partial r}=\frac{1}{r^{2}} \frac{\partial}{\partial r}\left(r^{2} k \frac{\partial T}{\partial r}\right)+2 \mu\left[\left(\frac{\partial v}{\partial r}\right)^{2}+\frac{v^{2}}{r^{2}}\right] \\
\rho \frac{\partial X_{i}}{\partial t}+\rho v \frac{\partial X_{i}}{\partial r}-\frac{1}{r^{2}} \frac{\partial}{\partial r}\left(r^{2} \rho D_{i} \frac{\partial X_{i}}{\partial r}\right)=0 ; \quad i=1,2, \ldots, n .
\end{gathered}
$$

As usual, $t$ is time, $r$ the radial coordinate, $\rho$ liquid density, $v$ liquid velocity, $p$ pressure, $T$ temperature and $X_{i}$ the liquid mass fraction of component $i$. The parameters $c_{l}, k, \mu$ and $D_{i}$ are respectively liquid heat capacity, thermal conductivity, viscosity and diffusivity coefficients. With respect to the initial conditions, uniform pressure, temperature and mass fraction of each specie are given at $t=0$,

$$
t=0: \quad p=p_{\infty}, T=T_{\infty}, X_{i}=X_{i \infty} .
$$

Moreover, the following Dirichlet conditions at infinity are considered:

$$
r \rightarrow \infty: \quad p=p_{\infty}, T=T_{\infty}, X_{i}=X_{i \infty} .
$$

The boundary conditions at the surface of the bubble, i.e. $r=a(t)$, are obtained from conservation and equilibrium equations at the bubble interface. To obtain the following equations, spatially uniform magnitudes (density, temperature, pressure and composition) are assumed inside the bubble, where gas velocities and spatial and temporal derivatives of the gas density and composition are neglected.

$$
\begin{gathered}
\rho_{g} \frac{\mathrm{d} a}{\mathrm{~d} t}+\rho\left(v-\frac{\mathrm{d} a}{\mathrm{~d} t}\right)=0, \\
\frac{2 \sigma}{a}=p_{g}-p+2 \mu \frac{\partial v}{\partial r}, \\
\frac{a}{3} \rho_{g} c_{v g} \frac{\mathrm{d} T_{s}}{\mathrm{~d} t}+l \rho_{g} \frac{\mathrm{d} a}{\mathrm{~d} t}-k \frac{\partial T}{\partial r}=0, \\
Y_{i s} \rho_{g} \frac{\mathrm{d} a}{\mathrm{~d} t}+\rho X_{i s}\left(v-\frac{\mathrm{d} a}{\mathrm{~d} t}\right)-\rho D_{i} \frac{\partial X_{i s}}{\partial r}=0 ; \quad i=1,2, \ldots, n, \\
f_{i}^{l}=f_{i}^{v} ; \quad i=1,2, \ldots, n .
\end{gathered}
$$


Here, the subscripts $g$ and $s$ distinguish magnitudes of gas inside the bubble and at the bubble interface (surface). Also, new variables $a$, bubble radius, $Y_{i}$, gas mass fraction of component $i$ and $f_{i}^{l}$ and $f_{i}{ }^{v}$, component fugacities of liquid and gas phases, are introduced. The parameters $\sigma, c_{v g}$ and $l$ are respectively surface tension, gas constant volume heat capacity and latent heat of evaporation.

\subsection{Non-dimensional model}

A first integral of the mass and momentum conservation equations leads to the Rayleigh-Plesset equation [9]:

$$
a \ddot{a}+\left[2-\frac{1}{2}\left(1-\frac{\rho_{g}}{\rho}\right)\right] \dot{a}^{2}=\frac{1}{\rho}\left[\frac{\Delta p_{g}-\frac{2 \sigma}{a}}{1-\frac{\rho_{g}}{\rho}}-\frac{4 \mu \dot{a}}{a}\right],
$$

where $\Delta p_{g}(t)=p_{g}(t)-p_{\infty}$ is the system supersaturation, which results from the chemical equilibrium at bubble interface (11). Moreover, to nondimensionalise the previous equation, the following variables are introduced:

$$
\hat{t}=\frac{k t}{\rho c_{l} a_{c}{ }^{2}}, \quad w=\frac{D_{T} \int_{0}^{t} a(s)^{4}}{a_{c}{ }^{6}}, \quad V_{b}=\frac{a^{3}}{a_{c}{ }^{3}}, \lambda=\left(\rho-\rho_{g}\right) a \dot{a} \frac{c_{l}}{k},
$$

which represent two different non-dimensional times (being $D_{T}$ the thermal diffusivity), the dimensionless volume and the dimensionless bubble growth velocity respectively. Moreover,

$$
\Psi_{S}=\frac{\Delta p_{g}}{\rho R M_{\infty}^{-1} T_{\infty}}
$$

is the dimensionless supersaturation, where $M_{\infty}$ is the molecular weight of the liquid and $R$ is the ideal gas constant. Thus, equation (12) is transformed into a system of three ordinary differential equations, with the corresponding initial conditions $a(0)=a_{c}, \dot{a}(0)=0$ :

$$
\begin{gathered}
\frac{\mathrm{d} V_{b}}{\mathrm{~d} w}=\frac{3}{2} \delta \frac{\lambda}{V_{b}} \\
\frac{\mathrm{d} \lambda}{\mathrm{d} w}=-\frac{\delta-1}{2} \frac{\lambda^{2}}{V_{b}^{2}}+\mathrm{P} \frac{\Psi_{s}}{V_{b}^{\frac{4}{3}}}-2 \operatorname{LaPr} \frac{1}{V_{b}^{\frac{5}{3}}}-4 \operatorname{Pr} \frac{\lambda}{V_{b}^{2}}, \\
\frac{\mathrm{d} \hat{t}}{\mathrm{~d} w}=\frac{1}{V_{b}^{4 / 3}}, \\
V_{b}(0)=1, \lambda(0)=0, \quad \hat{t}(0)=0,
\end{gathered}
$$

where the following dimensionless parameters have been introduced:

$$
\delta=\frac{2}{1-\frac{\rho_{g}}{\rho}}, \quad \operatorname{Pr}=\frac{\mu c_{l}}{k}, \quad \mathrm{P}=\frac{R M_{\infty}{ }^{-1} T_{\infty} a_{c}{ }^{2}}{D_{T}{ }^{2}}, \quad L a=\frac{\rho a_{c} \sigma}{\mu^{2}}
$$


The initial radius, $a_{c}$, is taken as the characteristic length. As the initial growth velocity is set to zero, the bubble only grows for initial radius larger than the critical radius, $a_{c}>\frac{2 \sigma}{\Delta p_{g}(0)}$.

Following [9] or [10], the resulting free boundary problem posed by the energy equation (3) and the boundary condition (9) can be transformed into a fixed boundary problem by using the new variable:

$$
\zeta=\frac{r^{3}-a(t)^{3}}{3 a_{c}{ }^{3}} .
$$

Furthermore, defining the dimensionless temperature $\Theta=\left(T-T_{\infty}\right) / T_{\infty}$, the dimensionless latent heat $\hat{l}=l /\left(T_{\infty} c_{l}\right)$ and the heat capacity ratio $\hat{c}_{v}=c_{v g} / c_{l}$, the boundary condition at the bubble surface reads:

$$
\left.\frac{\partial \Theta}{\partial \zeta}\right|_{\zeta=0}=\frac{\delta-2}{3 \delta}\left[\hat{l} \frac{\mathrm{d} V_{b}}{\mathrm{~d} w}+\hat{c}_{v} \frac{V_{b}}{3} \frac{\mathrm{d} \Theta_{s}}{\mathrm{~d} w}\right] .
$$

Both parameters $\hat{l}$ and $\hat{c}_{v}$ are of order unity, but $\hat{c}_{v}$ is usually smaller than $\hat{l}$. Moreover, assuming that temperature changes occur in a thin layer around the bubble, it can be inferred that $3 \zeta / V_{b} \ll 1$. Finally, making use of the initial condition and the boundary condition at infinity and defining $\epsilon=(\delta-2) / 2$, a good approximation of the thermal problem is:

$$
\begin{aligned}
& \frac{\partial \Theta}{\partial w}=\epsilon \frac{\lambda}{V_{b}} \frac{\partial \Theta}{\partial \zeta}+\frac{\partial^{2} \Theta}{\partial \zeta^{2}}, \quad w \in[0, \infty[, \quad \zeta \in[0, \infty[, \\
& \left.\frac{\partial \Theta}{\partial \zeta}\right|_{\zeta=0}=\epsilon \hat{l} \frac{\lambda}{V_{b}} ; \quad \Theta(\zeta, 0)=0 ; \quad \Theta(\infty, w) \rightarrow 0 .
\end{aligned}
$$

Making use of the same considerations as in the previous problem, the species continuity equations (4) and (10) take a similar form:

$$
\begin{gathered}
\frac{\partial \Delta X_{i}}{\partial w}=\epsilon \frac{\lambda}{V_{b}} \frac{\partial \Delta X_{i}}{\partial \zeta}+\frac{1}{L e_{i}} \frac{\partial^{2} \Delta X_{i}}{\partial \zeta^{2}}, w \in[0, \infty[, \quad \zeta \in[0, \infty[ \\
\left.\frac{\partial \Delta X_{i}}{\partial \zeta}\right|_{\zeta=0}=L e_{i} \epsilon \frac{\lambda}{V_{b}}\left(Y_{i}-X_{i s}\right) ; \Delta X_{i}(\zeta, 0)=0 ; \Delta X_{i}(\infty, w) \rightarrow 0 ; \quad i=1,2, \ldots, n .
\end{gathered}
$$

where the Lewis number, $L e_{i}=D_{T} / D_{i}$, and the incremental mass fraction, $\Delta X_{i}=X_{i}-X_{i \infty}$, of each component have been introduced.

\subsection{Chemical equilibrium}

The chemical equilibrium equation (11) allows to compute the equilibrium pressure at the interface and, as a consequence of that, the corresponding gas density and vapor composition can be obtained. In this work petroleum is modeled as a mixture of Methane $\left(\mathrm{CH}_{4}\right)$ and n-Decane $\left(\mathrm{CH}_{3}\left(\mathrm{CH}_{2}\right)_{8} \mathrm{CH}_{3}\right)$, and the Peng Robinson equation is used as equation of state [20]. Thus, it suffices to solve only one PDE for the species continuity equation, for example for the Methane (component 1 ). The value of $\Delta X_{2}$ is obtained using the relation $\sum_{i=1}^{n} X_{i}=1$, so $\Delta X_{2}=-\Delta X_{1}$.

To compute the coefficients of the Peng-Robinson equation, the molecular weight, the critical properties and the acentric factor of both species must be known [21,22]. With these data, along with the temperature and the composition of the liquid at the bubble surface, the saturation pressure and mass fractions of the gas components are obtained closing the previous 
system of equations: (11), (15)-(18), (22)-(23) and (24)-(25). It should be noticed that the chemical equilibrium is solved using an iterative method usually known as equilibrium flash computation. This process starts by using $p_{\infty}$ as an initial guess of the saturation pressure. To assure the convergence of the iterative process, the initial guess of the equilibrium constants is calculated using the approximate result given by Wilson equation [23].

\section{Results}

In this section, some representative results corresponding to the system of equations (11), (15)-(18), (22)-(23) and (24)(25) are presented. The spatial discretisation of the PDEs is made by means of Chebyshev rational polynomials of order $N=$ 15 collocated at Chebyshev-Gauss nodes (see [24, 25]). A $4^{\text {th }}$ order Runge Kutta scheme has been used for the time discretisation (time step $\Delta t=10^{-13} \mathrm{~s}$ ). Calculations have been done for a supersaturated mixture of Methane and $\mathrm{n}$-Decane (initial mole fractions $\hat{X}_{1 \infty}=0.55$ and $\hat{X}_{2 \infty}=0.45$ ) at an initial temperature of $375 \mathrm{~K}$ and three different supersaturations. Constitutive parameters have been obtained from several references: [26], [27], [28], [29] and [30], and, as a consequence, setting $a_{c}=10^{-8}$, the dimensionless parameters listed in Table 1 applies. It should be noticed that the latent heat has been approximated as $l=3,4 \cdot 10^{6} \mathrm{~J} / \mathrm{kg}$; this approximation will be justified in the next section.

Table 1: Dimensionless parameters.

\begin{tabular}{|c|c|c|c|}
\hline Parameter & Symbol & Definition & Value \\
\hline$L a$ & Laplace number & $\rho a_{c} \sigma / \mu^{2}$ & 0.722 \\
\hline$L e_{1} \mid L e_{2}$ & Lewis numbers & $D_{T} / D_{i}$ & $10.842 \mid 35.237$ \\
\hline$\hat{l}$ & $\begin{array}{c}\text { Dimensionless latent } \\
\text { heat }\end{array}$ & $l /\left(T_{\infty} c_{l}\right)$ & 0.479 \\
\hline$P r$ & Prandtl number & $\mu c_{l} / k$ & 2.457 \\
\hline$P$ & Pressure coefficient & $R M_{\infty}{ }^{-1} T_{\infty} a_{c}{ }^{2} / D_{T}{ }^{2}$ & 370.1935 \\
\hline$\epsilon$ & $\epsilon$ & $(\delta-2) / 2$ & 0.334 \\
\hline$\delta$ & $\delta$ & $2 /\left(1-\rho_{g} / \rho\right)$ & 2.669 \\
\hline
\end{tabular}




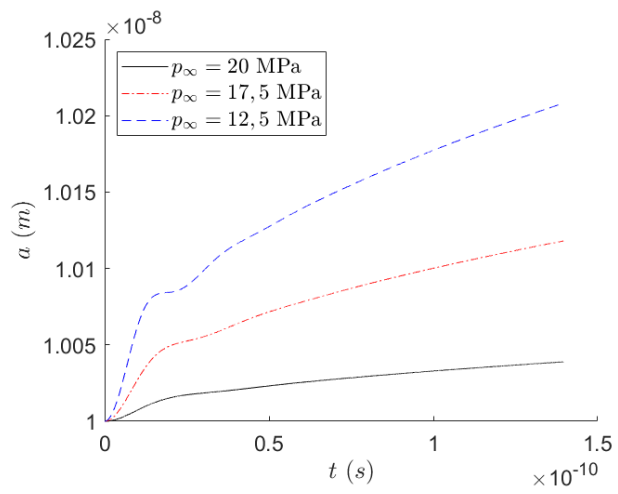

(a) Radius.

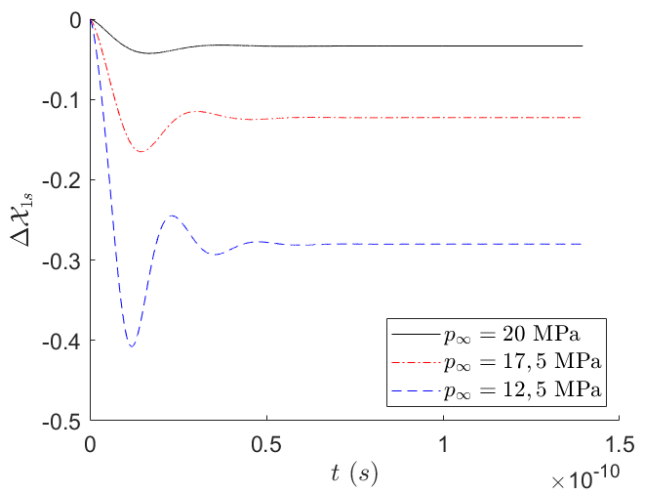

(c) Variation of mole fraction of Methane.

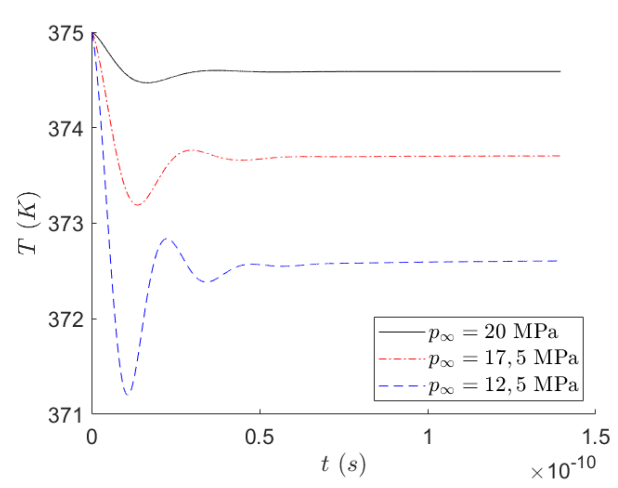

(b) Temperature.

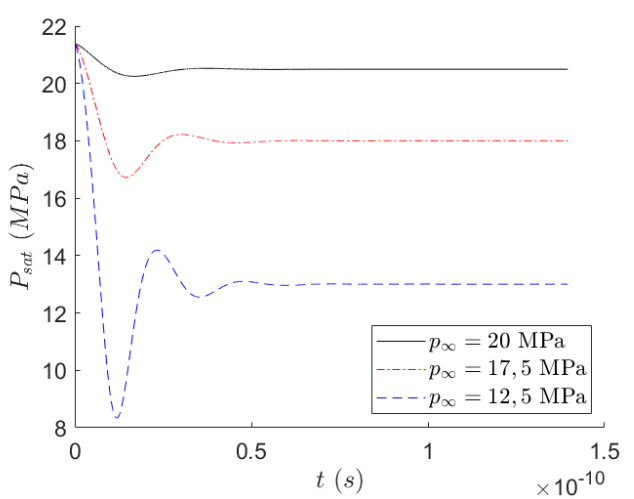

(d) Saturation pressure.

Fig. 1: Influence of initial supersaturation.

On the one hand, the obtained results (see Fig. 1) show that the bubble does not suffer important variations in the temperature and the radius evolutions. For example, the radius increases a $0.4 \%$ and the temperature decreases $0.4 \mathrm{~K}$ for the $p_{\infty}=20 \mathrm{MPa}$ test case. On the contrary, it can be seen in Fig. 1 (c) that the variation is bigger in the mole fraction of Methane which indicates that species diffusion is the dominant phenomenon in the bubble growth in petroleum, i.e., the decrease in the saturation pressure is due to the volatility of Methane. This is the expected result due to the Lewis numbers considered. When saturation pressure becomes constant, the value of the growth velocity falls and the expected diffusive growth of the bubble is reached. On the other hand, it should be noticed that higher supersaturations translate all the curves vertically but characteristic times remain unaffected. Species diffusion remains as the dominant phenomenon. It is also remarkable that the supersaturation reaches its minimum at the same time in all cases, and that species diffusion is faster as the initial supersaturation is higher.

\subsection{Influence of the latent heat}

In the previous section it was stated that an approximate value of the latent heat was used (we were not able to find the required values for the Methane and n-Decane mixture in the literature). There are models available for the computation of the latent heat (see for example [31] or [32]), however they are only valid for ideal conditions. Consequently, the values have been taken at standard conditions, and then the molar average of the mixture has been computed. A sensibility analysis to study the effect of this parameter has been performed (see Fig. 2). It is known that the latent heat decreases as the pressure and temperature conditions approach the critical point, where $l=0$, therefore the effect of decreasing its value has been studied. Fig. 2 shows that the only quantity that varies significantly is the temperature (the radius growth rate is not affected since species diffusion is dominant). 


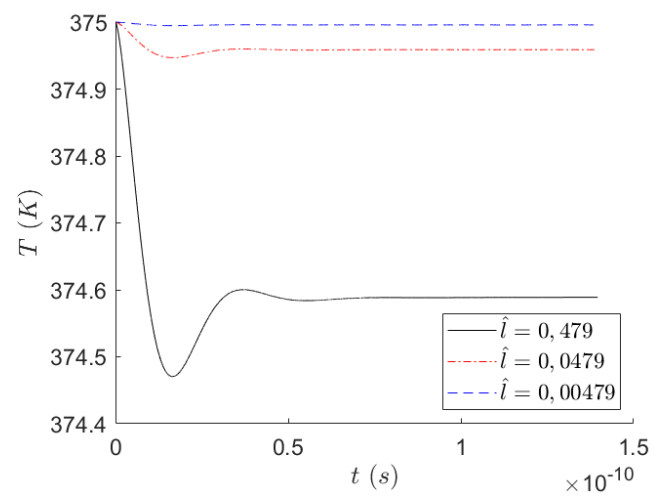

(a) Temperature.

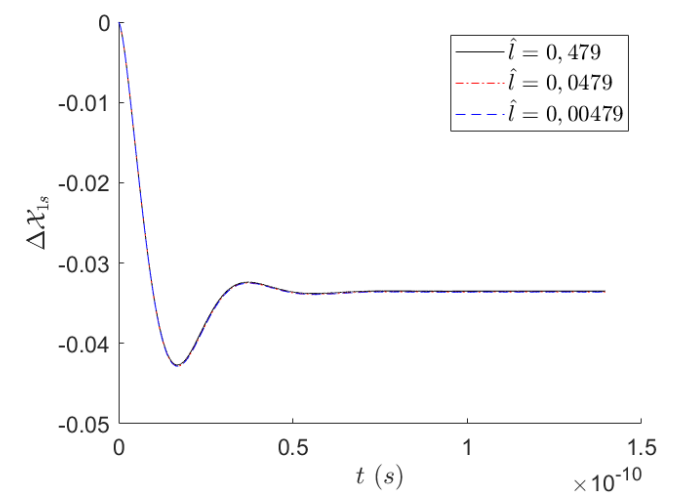

(b) Variation of mole fraction of Methane.

Fig. 2: Comparison with different values of the latent heat $\left(p_{\infty}=20 \mathrm{MPa}\right)$.

\subsection{Importance of the non-linear term}

The ratio between the gas and liquid densities is not negligible in the case considered. As a result, the non-linear term arising in equations of conservation of energy and species continuity should not be automatically neglected. Nevertheless, Fig. 3 shows that the effect of the non-linear term is not important for this particular problem. The reason behind this is that the aforementioned non-linear term is proportional to the bubble growth velocity, whose value is small in this particular problem.

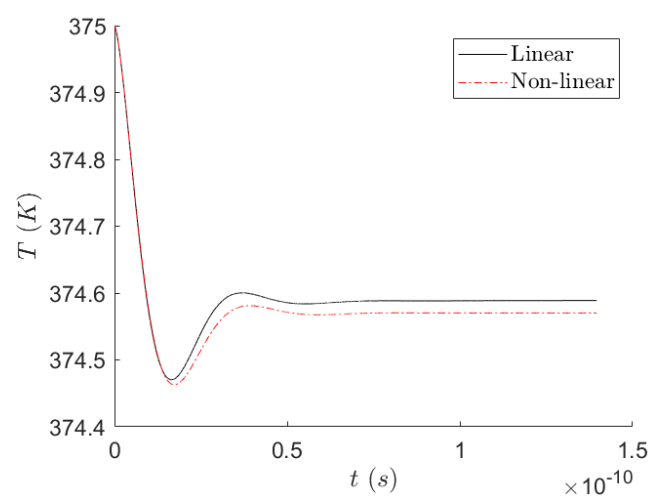

(a) Temperature.

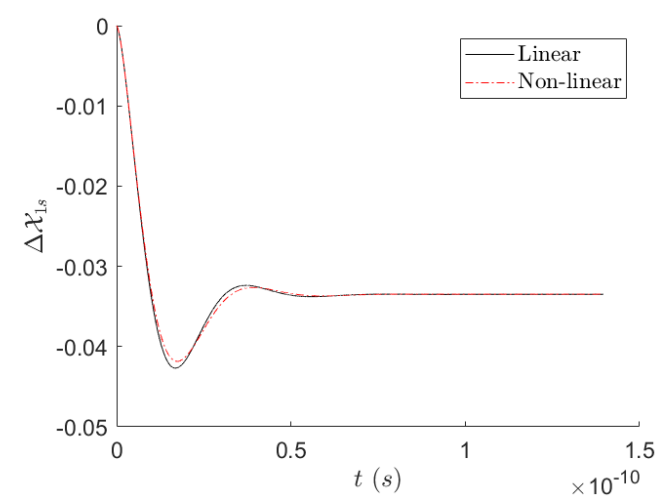

(b) Variation of mole fraction of Methane.

Fig. 3: Influence of the non-linear term $\left(p_{\infty}=20 \mathrm{MPa}\right)$.

\section{Conclusions}

In this work a model has been developed to study bubble growth in petroleum. It should be mentioned that the introduced model can be also applied to study other relevant problems in industry such as bubble growth in fire extinguishing multicomponent mixtures or refrigerants. The model has been used to study the bubble growth in a mixture of Methane and n-Decane, that can act as a simplified model for petroleum. We have shown that species diffusion controls the bubble growth rate. The supersaturation in the liquid rapidly decreases due to the volatility of Methane, until a stationary state is reached.

The effect of the initial supersaturation has been analysed. The conclusion is that higher saturations produce higher growth rates, caused by the increase of species diffusion. However, the characteristic times of each growth stage remain essentially unchanged. A sensibility analysis to the value of the latent heat has been performed. This has been mainly done because of the difficulty of obtaining precise values of this parameter. The conclusion is that this parameter only affects to 
the temperature of the bubble, which decreases proportionally to the value of the latent heat. Bubble growth is not affected, as it is driven by mass diffusion and not thermal diffusion in this problem.

Finally, the effect of the inclusion of a non-linear term in the model that results from the fact that the gas and liquid density ratio is not small has been analysed. The result is that the effect of this term is not important in this particular problem because of the small bubble growth velocity.

\section{Acknowledgments}

This work has been supported by Ministerio de Economía y Competitividad under the research grant TRA2015-67679C2-2-R.

\section{References}

[1] W. H. Besant, "A Treatise on Hydrostatics and Hydrodynamics," Cambridge University Press. 1859.

[2] L. Rayleigh, "On the Pressure Developed in a Liquid During the Collapse of a Spherical Cavity," in Philosophical Magazines vol. 34, pp. 94-98. 1917.

[3] M.S. Plesset, "The Dynamics of Cavitation Bubbles," in Journal of Applied Mechanics vol. 16, pp. 228-231. 1949.

[4] M. S. Plesset and S.A. Zwick, "The Growth of Vapour Bubbles in Superheated Liquids," in Journal of Applied Physics vol. 25, no. 4, pp. 493-500. 1954.

[5] G. Birkhoff, R.S. Margulies and W.A. Horning, "Spherical Bubble Growth," in Physics of Fluids vol. 1, no. 3, pp. 201204. 1959.

[6] D. E. Rosner and M. Epstein "Effects of Interface Kinetics, Capillarity and Solute Diffusion on Bubble Growth Rates in Highly Supersaturated Liquids," in Chemical Engineering Science vol. 27, pp. 69-88. 1972.

[7] B. B. Mikic, W. M. Rohsenow and P. Griffith, "On Bubble Growth Rates," in International Journal of Heat Mass Transfer vol. 13, pp.657-665. 1970.

[8] H. K. Forster and N. Zuber, "Growth of a Vapor Bubble in a Superheated Liquid," in Journal of Applied Physics vol. 25, no. 4, pp. 474. 1954.

[9] M. Dalle Donne and M. P. Ferranti (1975), "The Growth of Vapor Bubbles in Superheated Sodium," in International Journal of Mass and Heat Transfer vol. 18, pp. 477-493. 1975.

[10] M. S. Plesset and A. Prosperetti, "Vapour-bubble Growth in a Superheated Liquid," in Journal of Fluid Mechanics vol. 85, no. 2, pp. 349-368. 1978.

[11] M. S. Plesset and P. S. Epstein, "On the Stability of Gas Bubbles in Liquid-Gas Solutions," in Journal of Chemical Physics vol. 18, no. 11, pp. 1505-1509. 1950.

[12] M. Cable and D. J. Evans, "Spherically Symmetrical Diffusion-Controlled Growth or Dissolution of a Sphere," in Journal of Applied Physics vol. 38, no. 7, pp. 1999-2006. 1967.

[13] L. E. Scriven, "On the Dynamics of Phase Growth," in Chemical Engineering Science vol. 10, pp. 1-13. 1959.

[14] M. Li, Q. Zhu and G. Li, "Effect of Surface Tension and Viscosity on Bubble Growth of Single Mode Rayleigh-Taylor Instability," in Applied Mathematics and Mechanics vol. 37, no. 12, pp. 1607-1614. 2016.

[15] Y. Luo, J. Zhang, W. Li, E. Sokolova, Y. Li and W. J. Minkowycz, "Numerical Investigation of the Bubble Growth in Horizontal Rectangular Microchannels," in Num. Heat Transfer, Part A: Appl. vol. 71, no. 12, pp. 1175-1188. 2017.

[16] J. S. Amin, S. Tazikeh, S. Zendehboudi and I. Chazis, "New Modeling Strategies Evaluate Bubble Growth in Systems of Finite Extent: Energy and Environment Implications," in Industrial \& Engineering Chemistry Research vol. 57, no. 16, pp. 5680-5689. 1986.

[17] C. Redondo, G. Rubio and E. Valero, "On the efficiency of the IMPES method for two phase flow problems in porous media." in Journal of Petroleum Science and Engineering vol. 164, pp. 427-436. 2018.

[18] R. E. Terry and J. B. Rogers, "Applied Petroleum Reservoir Engineering," Prentice Hall. 2014.

[19] J. O. Hirschfelder, C. F. Curtiss and R. B. Bird, "Molecular Theory of Gases and Liquids," Wiley. 1954.

[20] D. Y. Peng and D. B. Robinson, "A New Two-Constant Equation of State," in Industrial \& Engineering Chemistry Fundamentals vol. 15, no. 1, pp. 59-64. 1976. 
[21] A. Danesh, "PVT and Phase Behaviour of Petroleum Reservoir Fluids," Elsevier Science \& Technology Books. 1998.

[22] H. Knapp and R. Döring, "Vapor-Liquid Equilibria for Mixtures of Low Boiling Substances," Dechema Chemistry Data Series, Volume VI, Part 1: Binary Systems. 1986.

[23] G. A. Wilson, “A Modified Redlich-Kwong EOS. Application to Physical Data Calculation,” in Annual Meeting of Chemical Engineers, paper 150. 1968.

[24] D. A. Kopriva, "Implementing Spectral Methods for Partial Differential Equations," Springer. 2009.

[25] G. Rubio et al, "The Estimation of Truncation Error by Tau-Estimation for Chebyshev Spectral Collocation Method," In Journal of Scientific Computing vol 57, no.1, pp. 146-173. 2013.

[26] T. S. Banipal, S. K. Garg and J. C. Ahluwalia, "Heat Capacities and Densities of Liquid n-Octane, n-Nonane, n-Decane, and n-Hexadecane at Temperatures from $318.15 \mathrm{~K}$ to $373.15 \mathrm{~K}$ at Pressures up to $10 \mathrm{MPa}$," in Journal of Chemical Thermodynamics vol. 23, pp. 923-931. 1991.

[27] D. K. Dysthe, A. H. Fuchs and B. Rousseau, "Prediction of Fluid Mixture Transport Properties by Molecular Dynamics," in International Journal of Thermophysics vol. 19, no. 2, pp. 437-448. 1998.

[28] C. Herdes, T. S. Totton and E. A. Müller, "Coarse Grained Force Field for the Molecular Simulation of Natural Gases and Condensates," in Fluid Phase Equilibria vol. 406, pp. 94-98. 2015.

[29] B. Knapstad, P. A. Skjolsvik and H. A. Øye, "Viscosity of the n-Decane - Methane System in the Liquid Phase," in Berichte der Bunsengesellschaft für physikalische Chemie, pp. 1156-1165. 1990.

[30] R. S. McDowell and F. H. Kruse, "Thermodynamic Functions of Methane," in Journal of Chemical and Engineering Data vol. 8, no. 4, pp. 547-548. 1963.

[31] J. E. Haggenmacher, "The Heat of Vaporization as a Function of Pressure and Temperature," in Journal of the American Chemical Society vol. 68, no. 8, pp. 1633-1634. 1946.

[32] K.M. Watson, "Thermodynamics of the Liquid State," in Industrial and Eng. Chem. vol. 35, no. 4, pp. 398-406. 1943. 